\title{
ORIGINAL ARTICLE \\ Similar but not the same: insights into the evolutionary history of paralogous sex-determining genes of the dwarf honey bee Apis florea
}

\author{
M Biewer ${ }^{1}$, S Lechner ${ }^{2}$ and M Hasselmann ${ }^{1}$ \\ Studying the fate of duplicated genes provides informative insight into the evolutionary plasticity of biological pathways to which \\ they belong. In the paralogous sex-determining genes complementary sex determiner (csd) and feminizer (fem) of honey bee \\ species (genus Apis), only heterozygous csd initiates female development. Here, the full-length coding sequences of the genes \\ csd and fem of the phylogenetically basal dwarf honey bee Apis florea are characterized. Compared with other Apis species, \\ remarkable evolutionary changes in the formation and localization of a protein-interacting (coiled-coil) motif and in the amino \\ acids coding for the csd characteristic hypervariable region (HVR) are observed. Furthermore, functionally different csd alleles \\ were isolated as genomic fragments from a random population sample. In the predicted potential specifying domain (PSD), \\ a high ratio of $\pi_{\mathrm{N}} / \pi_{\mathrm{S}}=1.6$ indicated positive selection, whereas signs of balancing selection, commonly found in other Apis \\ species, are missing. Low nucleotide diversity on synonymous and genome-wide, non-coding sites as well as site frequency \\ analyses indicated a strong impact of genetic drift in A. florea, likely linked to its biology. Along the evolutionary trajectory of \\ $\sim 30$ million years of csd evolution, episodic diversifying selection seems to have acted differently among distinct Apis branches. \\ Consistently low amino-acid differences within the PSD among pairs of functional heterozygous csd alleles indicate that the HVR \\ is the most important region for determining allele specificity. We propose that in the early history of the lineage-specific fem \\ duplication giving rise to $c s d$ in Apis, A. florea csd stands as a remarkable example for the plasticity of initial sex-determining \\ signals.
}

Heredity (2016) 116, 12-22; doi:10.1038/hdy.2015.60; published online 8 July 2015

\section{INTRODUCTION}

Duplicated genes may represent a rich source for novel gene function, although our current understanding of the different evolutionary fates leading to duplicated genes with modified gene functions remains incomplete (Innan and Kondrashov, 2010). The single-locus mechanism of complementary sex determination in honey bees provides a revealing example of how new gene functions can arise by duplication and limit the evolution of other genes (Hasselmann et al., 2008a, 2010). The primary signal of the sex-determining pathway in honey bees, the gene complementary sex determiner ( $c s d)$, originated by tandem gene duplication from its paralog gene feminizer ( fem) within the honey bee (Apis) lineage. The csd gene encodes an SR-type protein, harboring an arginine/serine-rich and a proline-rich domain. In addition to these domains and in contrast to fem, csd is characterized by the hypervariable region (HVR), forming repeated structures of species-specific amino acid motifs that are highly variable in length (Hasselmann et al, 2008b). Heterozygous csd is required to induce the female pathway by interacting with transformer 2 (Nissen et al, 2012), leading to a female-spliced fem transcript; this decision is maintained throughout the development by a positive feedback loop. Homozygous or hemizygous csd induces the male pathway, mediated by a truncated Fem protein, which results from an early stop codon in the male fem mRNA (Beye et al, 2003; Gempe et al, 2009). Fertilized eggs, homozygous at csd, develop into diploid males that have zero fitness, as they are consumed by worker bees at early embryonic stages (Woyke, 1963). Consequently, rare or newly evolved csd alleles that increase in frequency within a population rarely contribute to the formation of diploid males (Yokoyama and Nei, 1979). This rare-allele advantage or negative frequency-dependent selection as one form of balancing selection results in a prolonged persistence time for csd alleles that segregate in honey bee populations (Hasselmann and Beye, 2004; Hasselmann et al, 2008b).

The csd alleles of the different Apis species analyzed so far are on an average not older than the corresponding species split, although several csd alleles of Apis dorsata and A. mellifera could have predated speciation, as the divergence of allele pairs exceeds the mean interspecies divergence $\left(d_{\mathrm{S}}>0.14\right.$; Hasselmann et al., 2008b). Interestingly, in this previous study, no trans-specific alleles (alleles that are more closely related to an allele from another species than to other alleles from the same species) were detected (Hasselmann et al., 2008b). Trans-species alleles that can be maintained for $>30$ million years are frequently observed in other systems under balancing selection, such as in two well-studied cases: the major histocompatibility complex of vertebrates (Takahata, 1990) and the selfincompatibility S-locus of plants (Vekemans and Slatkin, 1994). In Apis, the relatively short average coalescence time of functional csd

${ }^{1}$ Department of Livestock Population Genomics, Institute of Animal Science, University of Hohenheim, Stuttgart, Germany and ${ }^{2}$ CeGaT GmbH - Center for Genomics and Transcriptomics, Tübingen, Germany

Correspondence: M Biewer, Department of Livestock Population Genomics, Institute of Animal Science, University of Hohenheim, Garbenstrasse 17, Stuttgart 70599, Germany. E-mail: matthias.biewer@uni-hohenheim.de

Received 11 December 2014; revised 1 May 2015; accepted 6 May 2015; published online 8 July 2015 
alleles (5-7 million years) can be best explained by a high allelic turnover rate, indicating small, long-term effective population sizes. Consequently, a strong historical impact of genetic drift as an evolutionary force might have affected csd allele evolution (Hasselmann et al, 2008b). A recent comprehensive evaluation of the dynamics of csd alleles in A. mellifera subpopulations showed that (i) the evolutionary rate giving rise to a new specificity is high and particularly driven by a HVR and that (ii) only few (4-5) amino acid substitutions are sufficient to give rise to a novel functional heterozygotic csd specificity (Lechner et al, 2014). In another study (Beye et al, 2013), the function and molecular evolution of 14 natural variants among 76 genotypes of $c s d$ were analyzed. The authors found evidence that at least five amino acid differences and length variation of the HVR are sufficient to induce female development. Interestingly, the rise of new specificities of csd likely evolves through a series of single mutations leading to incomplete penetrance of the advantageous phenotype (femaleness), supported by an evolutionary intermediate with only three amino-acid length differences.

Our knowledge about the evolutionary history of these paralogous genes is, however, still incomplete. Thus far, the csd gene has been intensively studied in three species of the genus Apis:A. mellifera, Apis cerana and A. dorsata. The csd alleles for each of the three Apis species are, on an average, $3 \%$ different at the amino acid level, excluding the HVR. In all three Apis species, support is given for a common target of balancing selection in $c s d$, the potential specifying domain (PSD) located in exons 6 and 7, based on high average diversity at synonymous $\left(\pi_{\mathrm{S}}\right)$ and nonsynonymous $\left(\pi_{\mathrm{N}}\right)$ sites (Hasselmann et al, 2008b). For A. florea and other Hymenoptera (for example, bumble bees), genome predictions have been used to describe fem and its orthologs (Schmieder et al, 2012). A putative csd ortholog has been reported in A. florea, showing exceptionally high-nucleotide diversity exceeding those described for any other Apis (Liu et al, 2011). However, their results are unexpected and may result from pseudogenic fragments with similarity to csd occurring in the genome, which has already given misleading assignments to putative csd alleles in other Apis species (Cho et al, 2006; Hasselmann et al, 2008b).

Previous evolutionary analyses showed that strong positive selection has acted on $c s d$ after the duplication event, leading to the divergence of A. mellifera, A. cerana and A. dorsata, accompanied by purifying selection for fem (Hasselmann et al, 2008a). Six amino acid residues associated with the fixation of nonsynonymous substitutions in the phylogenetic branch of $c s d$ prior to Apis species divergence gave rise to a predicted coiled-coil motif in the $c s d$ protein encoding proteinbinding properties (Lupas et al, 1991). In close sister lineages of corbiculate bees (for example, bumble and stingless bees), taking available genomic resources into account, no csd ortholog could be detected, indicating that the gene duplication of honey bee $c s d$ and fem occurred after the split of stingless, bumble and honey bees $(\sim 80$ million years ago), but before honey bee divergence, as an independent event (Koch et al., 2014). Recent phylogenetic analyses of molecular and morphological data place the open-nesting dwarf honey bee, $A$. florea, as the most basal clade in the phylogeny of the genus Apis (Lo et al, 2010). The estimated divergence time of this species from the rest of the genus Apis ranges from 29 to 33 million years, establishing A. florea as an ideal Apis species to gain broader insight into the evolutionary history and the early stage of the paralogous genes fem and csd (Ramirez et al, 2010).

In this study, we analyzed csd and fem gene sequences of $A$. florea to obtain a comprehensive view on the early evolutionary history of these important genes in bees. We identified the full-length coding sequence and genomic structure of both genes, obtained nucleotide polymorphism data from $A$. florea csd sequences, including nucleotide divergence of functional csd allele pairs in A. florea, and compared it against the genomic background. Using maximum-likelihood-based evolutionary models, we tested for evidence of positive selection within $c s d$ of the Apis phylogeny. Our data provide comparative insight into the evolutionary processes that have shaped the csd/fem complex in the past and show that modification of gene structures may have contributed to the astonishing diversity of primary signals in sex determination pathways.

\section{MATERIALS AND METHODS}

\section{Sequence data}

A. florea eggs ( $0-48 \mathrm{~h}, \sim 150$ eggs) and 20 adult female individuals were initially sampled in February 2009 from a colony in Thailand (Samut Songkram). Because of multiple mating of the queen, these samples have up to 15 different sources of chromosomes derived from as many different fathers (Palmer and Oldroyd, 2000). In a second sampling in February 2012, adult females were collected from 12 colonies in Chom Bueng District (Thailand) within a range of $1.6 \mathrm{~km}$ (Supplementary Table 1), and we used two individuals per colony to isolate the maximum-possible number of csd alleles (48 chromosomes). As shown in previous studies, high csd allelic variability is found within single colonies and localities of A. mellifera (Hasselmann and Beye, 2004; Lechner et al., 2014). Therefore, our sampling provides a substantial basis for the evaluation of $c s d$ allele diversity within A. florea. Sets of oligonucleotides were designed based on sequence information that was obtained from eight different $5^{\prime}$ and $3^{\prime}$ rapid amplification of complementary DNA (cDNA) ends (RACE) sequences. The following primers were developed: S-166 5'-CGGTTTCT CTAAGCATATAGGTGA-3' ${ }^{\prime}$ and S-158 5'-GTCAAGGCTGAGTAATAGTAT TAA-3' (used for full-length csd amplification); consfor 5'-GGTGATT TATACATTTGCAGGT-3' and A_rev3III 5'-ATTCAGTTCATTATTCA TTATTTGCA-3' (used for full-length fem amplification); S-156 5'-CTCC CGTTCTTCTTTTGATTATCACATT-3' and S-143 5'-CAGAAGAACGAT TACGACGGA GACGCG-3' (genomic fragment of csd exons 2 and 3); and S-176 5'-CATTTGACCCGCTAGTTGTCCAATCTCG-3' and S-177 5'-GTTGC AGTAGAGATAGAAATAGAGG-3' (genomic fragment of csd exons 6-9). Full-length $c s d$ and fem sequences were obtained from cDNA from pooled eggs of the 2009 sample. We noticed that our established protocol to identify different csd alleles (Hasselmann and Beye, 2004; Hasselmann et al, 2008b) using restriction patterns of full-length cDNA clones out of the pooled eggs failed for technical reasons (no detectable variation in restriction pattern). Samples of 20 female individuals (2009) were used to amplify genomic fragments of csd covering the corresponding regions of exons $2+3$ and 6-9 by PCR using highfidelity proofreading DNA polymerase (Phusion: Fermentas, Schwerte, Germany; Q5: New England Biolabs, Frankfurt a.M., Germany) according to the manufacturer's instructions. csd PCR fragments were cloned into pGEM-T vectors (Promega, Mannheim, Germany), and the positive clones were subjected to double-strand sequencing (GATC Biotech, Konstanz, Germany). From the 2012 samples, we obtained 32 csd exon 6-9 sequences, of which four were duplicates (identical sequences), which we removed in all subsequent analysis.

The genomic structures of $c s d$ and fem were identified through comparisons of full-length open reading frame cDNA sequences and amplified partial gene fragments from genomic DNA (this study) against the genomic resources available for A. florea (NCBI genome sequence accession numbers AEKZ01000001-AEKZ01019341, assembled as scaffolds GL575021-GL582965) using BLAST algorithms (blastn, tblastx) implemented in BeeBase (http:// hymenopteragenome.org/beebase/?q= blast). Amino acid sequences of $c s d$ and fem were deduced from corresponding cDNA sequences using EMBOSS transeq (http://www.ebi.uk/Tools/st/emboss_transeq).

Seven sets of oligonucleotides were developed to obtain nucleotide polymorphism from the genomic background of A. florea using seven presumably neutral evolving loci. Initially, we used pairs of oligonucleotides known to amplify neutral loci in A. mellifera and A. cerana (Beye et al, 2006). In those cases where amplification failed, we used alternative loci deduced from the genome sequences (GeneBank numbers AEKZ01002059.1, AEKZ01002102.1, 
AEKZ01002230.1; Supplementary Table 2). All loci were generated with highfidelity proofreading DNA polymerases and were subjected to direct sequencing.

Sequence data of csd alleles from A. mellifera, A. cerana and A. dorsata obtained in a previous study were used for comparative evolutionary analysis (Hasselmann et al, 2008b). For outgroup analyses, we used fem sequence data from Bombus terrestris (NCBI: NM_001280924.1), B. impatiens (NCBI: XM_003493748.1) and Melipona compressipes (NCBI: EU139305.1).

\section{Molecular evolutionary analysis of nucleotide and amino acid substitutions}

The $c s d$ sequences were aligned and edited as described elsewhere (Hasselmann and Beye, 2004). Sequence summary statistics in terms of haplotype diversity, number of segregating sites, estimates of nucleotide diversity and neutrality tests (Tajima's D (Tajima, 1989), Fu's F (Fu, 1997) and Fay and Wu's H (Fay and $\mathrm{Wu}, 2000)$ ) were computed in DnaSP 5.10 (Librado and Rozas, 2009). Combining tests for deviation from neutral evolution using the allele frequency spectrum can provide insight into the evolutionary processes within populations. A negative Tajima's D indicates population size expansion or selective sweeps, whereas a positive Tajima's D is associated with a recent bottleneck or overdominant selection. A negative Fu's $F_{s}$ statistic is evidence for an excess number of alleles, as expected from a population expansion or genetic hitchhiking. Positive values of $F_{s}$ are evidence for a deficiency of alleles and would indicate recent population bottleneck or overdominant selection. Fay and Wu's $\mathrm{H}$ statistic is most sensitive to a signal of a selective sweep, as reflected by the excess of high-frequency polymorphism that results in negative values. DnaSP 5.10 was also used to perform the Hudson-Kreitman-Aguadé test (Hudson et al, 1987) for neutral molecular evolution and test for recombination (four gamete test (Hudson and Kaplan, 1985)).

We used the best-fit substitution model application implemented in MEGA 5.1 (Tamura et al, 2011) to compare the available nucleotide substitution models and obtain the best description of the substitution pattern by maximum likelihood. The model with the lowest BIC scores (Bayesian Information Criterion) is considered to describe the substitution pattern the best. Nonuniformity of evolutionary rates among sites was modeled by using a discrete Gamma distribution $(+\mathrm{G})$ with five rate categories. By this approach, the Kimura-2 parameter model (with two categories and Gamma=1.63) was assigned as the best model, and trees were inferred from evolutionary distances by maximum likelihood.

Evolutionary sequence analyses to detect signs of selection in target genes were performed using the datamonkey web server interface (http://www. datamonkey.org), which accesses the HyPhy package (Kosakovsky Pond et al, 2005). The HyPhy package performs tests on evolutionary rate differences and signatures of selection using the Branch-Site REL model approach, which uses both branches and sites simultaneously for increased power and accuracy (Kosakovsky Pond et al, 2011).

\section{Structural analysis of amino acid composition and motifs}

The COIL program (Lupas et al, 1991; www.ch.embnet.org/software/ COILS form.html) was used to search the deduced full-length amino acid sequences of $c s d$ for predicted coiled-coil regions. The COIL program compares input sequences to a database of known coiled coils and derives a similarity score. The probability that the sequence will form a coiled-coil motif is obtained within the program by comparing the similarity score against the distribution of scores in lobular and coiled-coil proteins. InterPro scan (http://www.ebi.ac.uk/ interpro/) was used for additional sequence pattern analyses including coil motifs, domains and signatures in DNA and protein sequences.

\section{RESULTS}

\section{fem and csd gene analyses in A. florea}

We identified and isolated the sex-determining genes, fem and csd, from A. florea ( $A f-f e m$ and $A f$-csd) through reverse transcription PCR experiments using RNA from a pool of early $(0-48 \mathrm{~h})$ embryonic-stage eggs ( 150 eggs). The exon/intron structure of both genes is shown in Figure 1a and was deduced using the genome sequence information of A. florea. For the fem gene of A. mellifera, a female (encoding a full- length protein) and a male transcript (encoding a truncated protein) are known (Hasselmann et al, 2008a). We isolated the female splice form of Af-fem, consisting of 10 exons and encoding an open reading frame of 401 amino acids. Af-csd consists of nine exons, and the deduced open reading frame encodes $\sim 408$ amino acids (depending on length variation within the HVR). In A. florea, the genomic region covered by exons encoding the fem gene is $7 \mathrm{~kb}$ long, whereas csd spans a genomic region of $8.7 \mathrm{~kb}$ located $3.5 \mathrm{~kb}$ downstream of fem. This is in contrast to A. mellifera, for which the distance between fem and $c s d$ is approximately four times as large $(\sim 12 \mathrm{~kb})$. The amino acid sequences of $A f-f e m$ and $A f-c s d$ deduced from the full-length cDNA indicated a similar domain structure as described previously for Csd and Fem protein in other Apis species (Hasselmann et al, 2008a, Figure 1b). Csd protein harbors a arginine-serine (RS)-rich (red) and proline (P)-rich (blue) region corresponding to exons 6-9, which are flanking a HVR (green). Fem protein is characterized by two RS-rich domains and a P-rich domain and lacks a HVR.

The small physical distance of $3.5 \mathrm{~kb}$ between $A f-f e m$ and Af-csd located on a single scaffold segment provides strong support for the existence of a single sex determination locus in A. florea, which is consistent with the genome structure described for A. mellifera. Nucleotide sequence analyses on full-length open reading frames of both genes using BLAST against available genome sequence resources confirmed the instances as single-copy genes of each, verified by highly significant single matches (score: $166, e$-value $10^{-169}$ ).

\section{Differences between the A. florea csd protein and those of other Apis species}

To elucidate the functional characteristics of A. florea csd alleles, we further analyzed the Csd protein sequence. A striking characteristic of all $c s d$ genes is the HVR. This region has been shown to be highly variable in length and composition among csd alleles within the same Apis species (Beye et al, 2003; Hasselmann et al, 2008b). The amino acid residues flanking the HVR are highly conserved within a single Apis species. A cross-species comparison reveals that several of those amino acids are also conserved, allowing an unambiguous alignment shown in Figure 1b. Remarkably, the HVR of A. florea consists only of asparagines together with some single serine residues (Figures $1 \mathrm{~b}$ and 2a). This structure contrasts with the recurrence of characteristic motifs found in other Apis species $\left(\mathrm{N}_{1-5} \mathrm{Y}\right.$ for A. mellifera, $\mathrm{KHYN}_{1-}$ ${ }_{4} \mathrm{KH}$ for A. cerana and A. dorsata; Figure 1b, Hasselmann et al, 2008b). By isolating both csd alleles from A. florea females, we were able to compare pairs of functional alleles forming a heterozygous $c s d$ gene. In all the pairs of heterozygous $c s d$ alleles analyzed, the HVR between the two chromosomes differed in length (1-18 amino acids of length difference) and composition (serine and asparagine residues; Figure 2a). To gain additional insight into putative functional differences of $A$. florea Csd compared with other known Csd proteins in Apis, we searched for coiled-coil motifs in A. florea. Coiled-coil motifs are known to be involved in protein-protein interactions (Lupas et al, 1991) and have been proposed to contribute to the interaction between Csd proteins (Hasselmann et al, 2008a). First, we focused on the region in which the coiled-coil motif is predicted for other Apis species (Figure 1a, marked with an asterisk). In this particular region, we found among the six amino acids in Csd that are conserved within the motif of A. mellifera, cerana and dorsata (Figure 2b, black box) one amino acid residue that was different in A. florea (Valin (V) instead of glutamate (E)), leading to a strongly decreased probability for a coiled-coil formation in A. florea ( $>98 \%$ for A. mellifera, cerana and dorsata vs $<92 \%$ for A. florea). Interestingly, a highly significant prediction of a coiled-coil motif was found in 
a
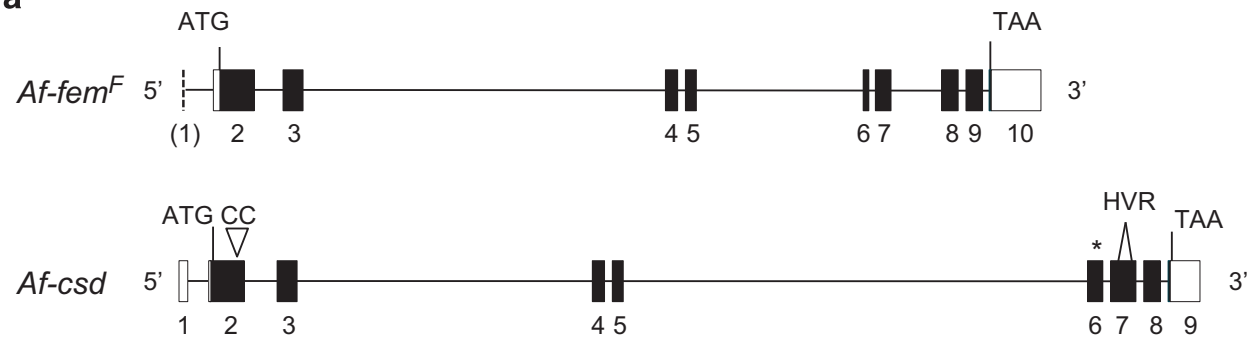

$1 \mathrm{~kb}$

b
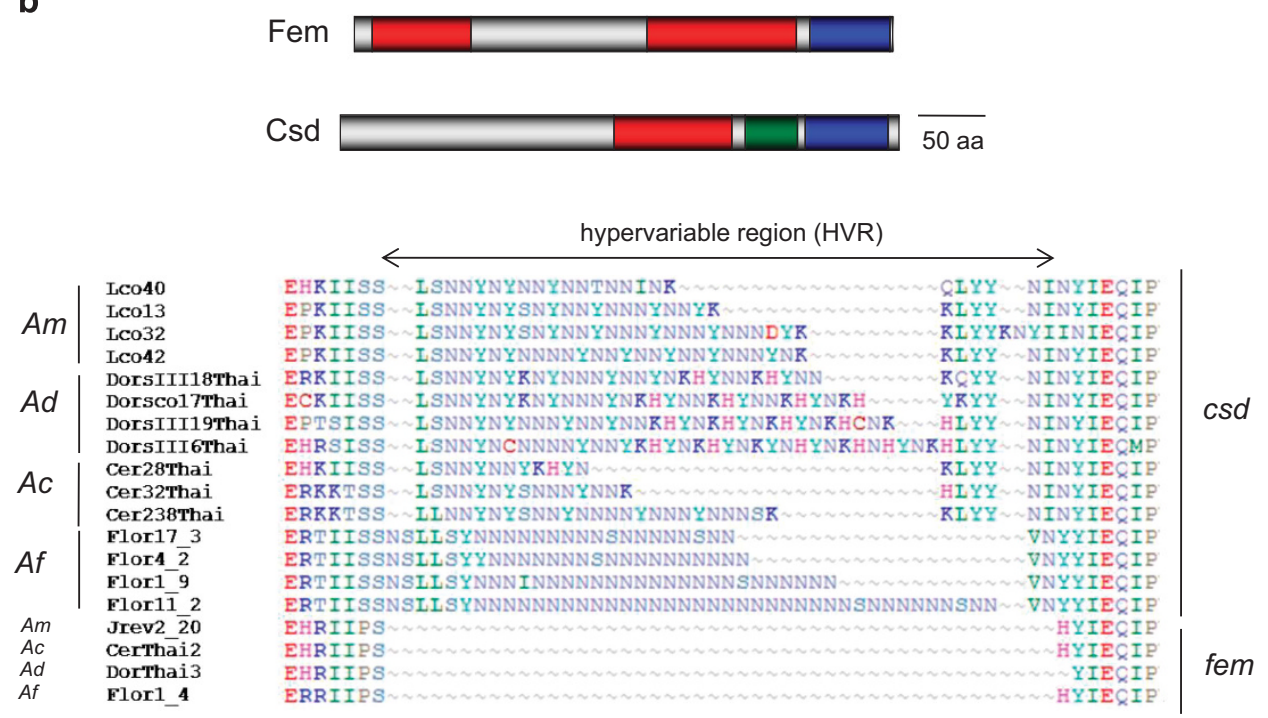

Figure 1 Genomic structure of the fem (Af-fem ${ }^{F}$, female splice variant) and csd (Af-csd) genes in Apis florea, domain diagram of Csd and Fem and conceptual translations spanning the hypervariable region (HVR) of four honey bee species. (a) Coding exons are marked in black; untranslated regions are in white. Translational start and stop sites are indicated. In the csd gene, the position of the HVR is marked (exon 7). * indicates the position of the predicted coiled-coil motif in A. mellifera, A. cerana, A. dorsata, but missing in A. florea (see Figure 2b). In A. florea, a coiled-coil motif is predicted in exon 2 (marked as CC). (b) Protein domains of Csd and Fem (above): arginine-serine domains are indicated in red, the HVR in green and the proline-rich region in blue. Below: conceptual alignment of deduced amino acids residues of the HVR in csd and the corresponding region of fem. Am, A. mellifera; Ad, A. dorsata; Ac, A. cerana; Af, A. florea.

the N-terminal region (position aa 46-70; Figure 1a, marked with a triangle; Figure 2c) located $\sim 240$ amino acids upstream compared with the region analyzed first. No coiled-coil motif has been predicted within the $\mathrm{N}$-terminal region of Csd in any other Apis species studied, likely due to the absence of two amino acids, displayed as gaps in the alignment, and a single amino acid substitution of leucine (L) for arginine ( $\mathrm{R}$; Figure $2 \mathrm{c}$, black box). In addition to this finding, one $\mathrm{N}$-terminal coiled-coil motif at the corresponding position to Csd (aa 46-70) was found for Fem in A. florea, but not in the other three Apis species (98.9\% probability for A. florea vs $<65 \%$ for A. mellifera/A. cerana/A. dorsata; Figure 2c, black box), accompanied by a lineage-specific deletion of two amino acids. No such deletion is found in any other Hymenopteran non-Apis outgroup species we tested (B. terrestris, B. impatiens, M. compressipes), and no coiled-coil motif is predicted for these species (data not shown). Consequently, the additional coiled-coil motifs for Af-Csd and Af-Fem in A. florea suggest an altered position for protein-protein interaction among these molecules in the process of sex determination compared with other Apis species. In the early phase of the evolution of paralogous genes within the Apis lineage, we observed a transition in primary structure resulting in modifications of protein structure and function.
Reduced variability and evolutionarily young csd alleles in A. florea We obtained nucleotide polymorphism data from exons 6-9 of Af-csd from females of 1 colony sampled in 2009 and 12 colonies sampled in 2012 to analyze the molecular evolutionary history of the csd alleles in A. florea (Table 1). We compared these data with the nucleotide polymorphisms of seven non-coding, unlinked loci obtained from 12 individuals of different colonies examined in 2012 (Table 2), as well as with data on $A f-c s d$ exons $2+3$ and Af-fem exons 2-7 obtained from a subsample of these colonies (Supplementary Table 3 ).

Nucleotide diversity $(\pi)$ in exons $6+7$ of $A f$-csd significantly exceeded the diversity in exons $2+3$ of $A f-c s d$ for both data sets $\left(\pi_{\mathrm{ex} 2+3}=0.00248\right.$ vs $\pi_{\mathrm{ex} 6+7}=0.00579$ (2009), $Z$-test, $P<0.001$ and $\pi_{\mathrm{ex} 6}$ $+7=0.00675$ (2012), $P<0.001)$. Nucleotide diversity in exons $6+7$ of Af-csd also exceeded the diversity in Af-fem $\left(\pi_{\text {ex } 6+7 \mathrm{fem}}=0.00135\right.$; $P<0.001$ (2009) and $P<0.001$ (2012)). Remarkably, intron diversity within Af-csd exceeded those for synonymous sites $\left(\pi_{\text {intron_combined }}=\right.$ 0.0094 vs $\left.\pi_{\text {s_combinedPSD }}=0.0035, P<0.05\right)$, which are both surprisingly low when compared with the corresponding values from other Apis species (for example, A. mellifera $\pi_{\text {syno_PSD }}=0.07-0.09$, Lechner et al. (2014)).

A comparison of nonsynonymous nucleotide diversity $\left(\pi_{\mathrm{n}}\right)$ between the two exonic regions of the csd gene showed a higher $\pi_{\mathrm{n}}$ for exons 6 


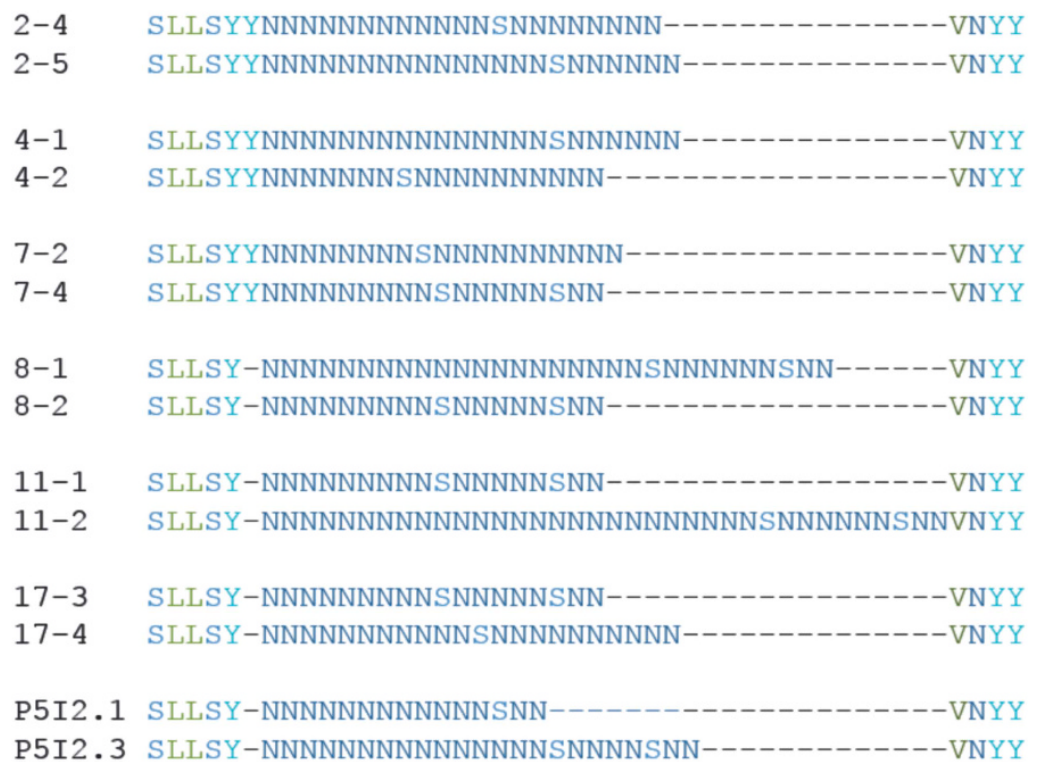

b

Coiled-Coil

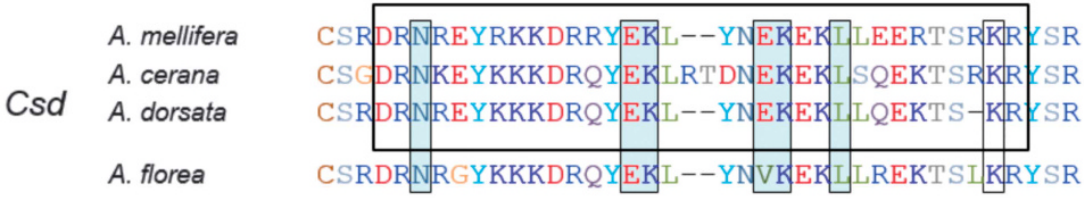

$\begin{array}{ll}\text { A. mellifera } & \text { CSRDRSREYKKKDRRYDQL--HNVEEKHLRERTSRRRYSR } \\ \text { Fem A. cerana } & \text { CSRDR.SREYKKKDRRYDQL--HNVEEKHLRERTSRRRYSR } \\ \text { A. dorsata } & \text { CSRDR.SREYKKKDRRYDQL--HNVEEKHLRERTNRRRYSR } \\ \text { A. florea } & \text { CSRDRSREYKKN-RRYEP--HNVEEKHLRERTSRRRYSR }\end{array}$

C

\section{Coiled-Coil}

$\begin{array}{ll}\text { A. mellifera } & \text { WLIQQEREREHQRLMKKMILEYELRRIREIEKLGS } \\ \text { Csd cerana } & \text { WMIQQEREREHEKLKKKMILEYELRRAREKKLALS } \\ \text { A. dorsata } & \text { WMIQQEREREYEKLKKKRILEYELRRAREKKL-L- } \\ \text { A. florea } & \text { WMIQEELEREHERLKKKMILEYE-TRAREKKLALS }\end{array}$

$\begin{array}{ll}\text { A. mellifera } & \text { WMIQQEREREHERLKKKMILEYELRRAREKK--LS } \\ \text { Fem A. cerana } & \text { WMIQQEREREHERLKKKMILEYELRRAREKKLALS } \\ \text { A. dorsata } & \text { WMIQQEREREHERLKKKMILEYELRRAREKKLALS } \\ \text { A. florea } & \text { WMIQQEREREHERLKKKMILEYE--RAREKKLALS }\end{array}$

Figure 2 Characteristics of Csd and Fem protein in A. florea. (a) The aligned conceptual translation of the hypervariable region (HVR) between seven pairs of functionally different csd alleles of females is shown. The HVR encodes a succession of asparagine residues (N) and single serine residues (S). (b) Deduced amino acid sequence encoding the region of the predicted coiled-coil motif, framed in black. For A. mellifera, $A$. cerana and $A$. dorsata csd, the prediction is based on the six amino acids in gray shaded boxes. A. florea differs in one position compared to the other species and does not form a coiled-coil motif (unframed). The homologous region for fem is placed below for comparison, with the corresponding amino acids marked. (c) Deduced amino acid sequence encoding the region of the predicted $\mathrm{N}$-terminal coiled-coil motif for $A$. florea framed in black. Only one amino acid change and a two amino acid difference shown as a gap (shaded boxes) seem sufficient for the formation in Csd and Fem in A. florea, whereas this is not found in the other Apis species (unframed).

+7 than exons $2+3(P<0.05)$ in both the 2009 and 2012 samples. We noted that the diversity of nonsynonymous sites exceed those of the synonymous sites (Fisher's exact test, $P<0.01$ ) in the region of exons 6 +7 , which is more pronounced in the 2009 data than in the 2012 data
(2009: $\pi_{\mathrm{n}} / \pi_{\mathrm{s}}=5.71 ; 2012: \pi_{\mathrm{n}} / \pi_{\mathrm{s}}=1.17$ ). These findings are in agreement with our previous results, establishing the region of exon $6+7$ as a common target of balancing selection and predicting the specifying domain (csd-PSD) in the Apis species. The ratio $\pi_{\mathrm{n}} / \pi_{\mathrm{s}}=1.6$ of the 
Table 1 Summary statistic of csd sequences of Apis florea

\begin{tabular}{|c|c|c|c|c|c|c|c|c|c|c|c|c|c|c|}
\hline & \multirow[t]{2}{*}{$\mathrm{N}$} & \multirow[t]{2}{*}{$\mathrm{H}_{d}$} & \multirow[t]{2}{*}{$n$} & \multirow[t]{2}{*}{ S } & \multirow[t]{2}{*}{$\pi$} & \multicolumn{2}{|l|}{ csd } & \multirow[t]{2}{*}{$\pi_{n}$} & \multirow[t]{2}{*}{$\pi_{n} / \pi_{S}$} & \multirow[t]{2}{*}{$D$} & \multirow[t]{2}{*}{$\mathrm{D}_{s}$} & \multirow[t]{2}{*}{$\mathrm{D}_{n}$} & \multirow[t]{2}{*}{$\mathrm{F}_{\mathrm{s}}$} & \multirow[t]{2}{*}{$\mathrm{H}$} \\
\hline & & & & & & $\Theta_{W}$ & $\pi_{S}$ & & & & & & & \\
\hline \multicolumn{15}{|l|}{ Sample 2009} \\
\hline Exon 6-9 & 23 & 0.68 & 438 & 15 & $4.78 \pm 0.1$ & $9.28 \pm 3.7$ & 2.42 & 5.61 & 2.32 & $-1.73^{*}$ & -1.73 & -1.51 & $-3.81^{*}$ & $-3.04^{*}$ \\
\hline PSD $(6+7)$ & 23 & 0.58 & 273 & 10 & $5.79 \pm 1.4$ & $9.92 \pm 4.4$ & 1.28 & 7.31 & 5.71 & -1.4 & -1.16 & -1.28 & -1.66 & -1.38 \\
\hline Intron & 23 & 0.79 & 201 & 7 & $9.56 \pm 1.6$ & $9.44 \pm 4.5$ & - & - & - & 0.04 & - & - & -1.99 & - \\
\hline \multicolumn{15}{|l|}{ Sample 2012} \\
\hline Exon 6-9 & 28 & 0.88 & 444 & 9 & $4.90 \pm 0.5$ & $5.21 \pm 2.3$ & 2.83 & 5.71 & 2.02 & -0.14 & 0.29 & -0.23 & $-6.57^{* *}$ & $-3.15^{*}$ \\
\hline PSD $(6+7)$ & 28 & 0.85 & 279 & 7 & $6.75 \pm 0.7$ & $6.45 \pm 3.1$ & 4.52 & 5.29 & 1.17 & 0.14 & 0.29 & -0.39 & $-3.65^{*}$ & -1.3 \\
\hline Intron & 28 & 0.69 & 201 & 5 & $8.1 \pm 0.9$ & $6.39 \pm 3.4$ & - & & & 0.75 & - & - & 0.61 & - \\
\hline \multicolumn{15}{|l|}{ comb. data } \\
\hline Exon 6-9 & 51 & 0.84 & 423 & 19 & $5.15 \pm 0.6$ & $9.98 \pm 3.5$ & 2.87 & 5.95 & 2.07 & $-1.53^{*}$ & $-1.50 *$ & -1.33 & $-11.97^{* *}$ & $-5.66^{* *}$ \\
\hline PSD $(6+7)$ & 51 & 0.78 & 258 & 13 & $6.87 \pm 0.8$ & $11.20 \pm 4.3$ & 3.45 & 5.46 & 1.58 & -1.16 & -0.9 & $-1.50 *$ & $-3.34^{*}$ & -1.9 \\
\hline Intron & 51 & 0.77 & 200 & 8 & $9.36 \pm 0.9$ & $8.89 \pm 3.9$ & - & - & - & 0.14 & - & - & -1.39 & - \\
\hline
\end{tabular}

Abbreviations: D, Tajima's D; $F_{s}$, Fu' $s F_{s} ; H$, Fay and Wu's $H$ statistic; $H_{d}$, haplotype diversity; $N$, sequence number; $n$, number of nucleotides; $S$, number of segregating sites; $\pi$, average pairwise nucleotide diversity for all; $s$ and $n$, synonymous and nonsynonymous sites; $\Theta_{w}$, Watterson's Theta.

Values for $\pi$ and $\theta_{W}$ are given $\times 1000$. PSD: potential specifying domain. Intron sequences are from csd region exon $6-9$

Values for $\pi$ and $\theta_{\mathrm{w}}$ ar
$* P<0.05, * * P<0.01$

Table 2 Summary statistic of unlinked, neutral loci sequences of Apis florea

\begin{tabular}{|c|c|c|c|c|c|c|c|c|c|}
\hline & \multirow[t]{2}{*}{$\mathrm{N}$} & \multirow[t]{2}{*}{$\mathrm{H}_{d}$} & \multirow[t]{2}{*}{$n$} & \multirow[t]{2}{*}{ S } & \multicolumn{2}{|c|}{ Neutral loci } & \multirow[t]{2}{*}{$D$} & \multirow[t]{2}{*}{$\mathrm{F}_{\mathrm{S}}$} & \multirow[t]{2}{*}{$\mathrm{H}$} \\
\hline & & & & & $\pi$ & $\Theta_{W}$ & & & \\
\hline Locus 2 & 12 & 0.17 & 346 & 1 & $0.48 \pm 0.4$ & $0.96 \pm 0.9$ & -1.14 & -0.48 & 0.15 \\
\hline Locus 4 & 12 & 0.62 & 223 & 2 & $3.19 \pm 0.8$ & $2.97 \pm 2.3$ & 0.22 & 0.03 & 0.51 \\
\hline Locus 8 & 11 & 0.82 & 263 & 7 & $8.16 \pm 1.6$ & $9.09 \pm 4.8$ & -0.42 & $-2.65^{*}$ & 0.09 \\
\hline Locus 9 & 12 & 0.41 & 195 & 1 & $2.10 \pm 0.7$ & $1.70 \pm 1.7$ & 0.54 & 0.74 & 0.27 \\
\hline Locus 11 & 12 & 0.82 & 316 & 5 & $4.22 \pm 0.9$ & $5.24 \pm 2.9$ & -0.72 & $-2.46^{*}$ & - \\
\hline Locus 12 & 12 & 0.8 & 317 & 5 & $4.35 \pm 0.9$ & $5.22 \pm 2.9$ & -0.62 & -1.11 & - \\
\hline Locus 13 & 12 & 0.65 & 544 & 3 & $1.42 \pm 0.4$ & $1.83 \pm 1.2$ & -0.73 & -1.17 & - \\
\hline Average & & 0.61 & & 3.4 & 3.42 & - & - & - & - \\
\hline
\end{tabular}

Abbreviations: D, Tajima's D; $F_{s}$, Fu' s $F_{s} ; H$, Fay and Wu's $H$ statistic; $H_{d}$, haplotype diversity; $N$, sequence number; $n$, number of nucleotides; $S$, number of segregating sites; $\pi$, average pairwise nucleotide diversity for all; $\Theta_{w}$, Watterson's Theta.

Values for $\pi$ and $\theta_{\mathrm{w}}$ are given $\times 1000$

${ }^{*} P<0.05$.

combined data set is still remarkably higher in A. florea-PSD when compared with the three other Apis species with ratios of $\pi_{\mathrm{n}} / \pi_{\mathrm{s}}<1$, ranging from 0.6 to 0.95 for $c s d$-PSD, due to an accumulation of synonymous polymorphism over time in obviously older alleles (Hasselmann et al, 2008b). Consequently, as we observe $\pi_{\mathrm{n}} / \pi_{\mathrm{s}}$ ratios $>1$ for A. florea, we conclude that csd alleles in A. florea populations are comparatively young.

We used polymorphism data from seven non-coding, presumably neutrally evolving loci, to gain insight into the genome-wide nucleotide diversity of $A$. florea. On an average, $\pi_{\text {genome }}$ is 0.00341 (ranging from 0.00048 to 0.00816 , Table 2), which is almost identical to the diversity of synonymous sites in Aflor-csd-PSD $\left(\pi_{\text {syno_combinedPSD }}=\right.$ 0.00345 ), indicating the genetic drift as the dominant evolutionary force affecting csd allele evolution in A. florea.

To gain insight into population dynamics, we determined whether the observed nucleotide polymorphisms might be affected by demographic effects by analyzing their frequency spectrum. Population growth is known to result in a biased frequency spectrum of polymorphism, which would be reflected in negative values of Tajima's D. For all data sets, except csd intron sequences, Tajima's
D-values were negative or close to zero. The values for synonymous sites at $c s d$ exons 6-9 (combined data: $D_{\mathrm{s}}=-1.50$ ), nonsynonymous sites at $c s d$-PSD $\left(D_{\mathrm{n}}=-1.50\right.$, Table 1$)$ and overall $(D=-1.53)$ were significant $(P<0.05)$, which might indicate an expanding population or enhanced fixation of nonsynonymous substitutions within the csd gene (selective sweep). We applied Fu's $\mathrm{F}_{\mathrm{s}}$ statistic, which is known to be particularly sensitive for recent population expansions, based on the number of haplotypes observed in the sample (Fu, 1997). Fu's $F_{s}$ was significant for csd exons 6-9 $(P<0.01)$ and csd-PSD $(P<0.05$, combined data). These values support Tajima's test and led us to discriminate the potential effect of population expansion from an alternative scenario, which would be a selective sweep, by applying Fay and Wu's H-statistic (Fay and Wu, 2000). Fay and Wu's H-statistic was significant for csd exons 6-9 $(H=-5.66, P<0.01)$, but marginally insignificant for csd-PSD $(H=-1.9)$. Combined, our results suggest that the observed $c s d$ alleles belong to an expanding population after a bottleneck event, with an advantage for new alleles, and a fixation of haplotypes that have recently arisen.

The analyses of the non-coding loci revealed that Tajima's D values were close to zero in all loci, which is expected from neutral evolution 
following mutation-drift equilibrium. To test for the signs of population expansion in neutral loci, we applied Fu's $\mathrm{F}_{\mathrm{s}}$-test. In two of the seven loci, we observed significant values for $F_{s}(P<0.05)$, providing weak indications of an expanding population after a bottleneck in these populations, as was found in the csd samples. Fay and Wu's H-statistic was close to zero, as expected for these unlinked neutral loci.

To relate our findings to the evolutionary time, which would be necessary for the rise of new csd specificities within A. florea, we followed the approach based on the neutral mutation rate per site per generation $\left(\mu=2.16 \times 10^{-9}\right.$; Lechner et al, 2014). The number of generations per year in A. florea can vary from one to three, depending on the reproduction cycles linked to swarming activities that can be induced by variation in food resources and climatic conditions (Hepburn, 2011). Using a model for neutral evolving nucleotides, $\pi_{\mathrm{s}}=4 N \mu$ (using $\pi_{\mathrm{s}}=0.00345$ ), with the above average neutral mutation rate per site per generation, we obtain $N=4 \times 10^{5}$ generations to accumulate these differences. Under this assumption, the expected number of nonsynonymous substitutions within the PSD region is 1 ( $N \mu \times$ the number of nonsynonymous sites (209)), which equals the number of amino acid changes (1-3) found among $A$. florea csd alleles using the corresponding $\pi_{\mathrm{n}}$ values ranging from $\pi_{\mathrm{n}}=0.0048$ to 0.0145 . Based on this result, we calculated an origination rate $(u)$ of new csd alleles per gene per generation of about $u=4.5 \times 10^{-7}$. If we assume that two amino acid differences within the PSD are needed to generate a new specificity, our results suggest the formation of a new csd allele in $A$. florea every 400 000-800 000 generations. As mentioned before, A. florea may swarm (and reproduce) up to three times a year. Consequently, the time necessary for a new csd specificity to arise may range from 130000 (three swarmings) to 800000 (one swarming) years.

To exclude the alternative scenario of an elevated mutation rate in A. florea csd-PSD, indicated by the high ratios found for $\pi_{\mathrm{n}} / \pi_{\mathrm{s}}$, we performed the Hudson-Kreitman-Aguadé test. We compared the pattern of polymorphism of $c s d$-PSD and the unlinked neutral locus 4 within A. florea to the divergence data from A. dorsata. The HudsonKreitman-Aguadé test detected no significant deviation in the number of synonymous sites from a constant ratio of polymorphism to divergence in csd-PSD and locus 4. However, we are aware of the fact that the Hudson-Kreitman-Aguadé test is only valid in the absence of recombination within and free recombination between the loci, which we cannot completely rule out to be the case here.

To obtain further insight into the divergence of the PSD among csd alleles, we calculated the nucleotide differences for 14 pairs of functionally different $c s d$ alleles separately. The pairwise calculations of synonymous and nonsynonymous substitutions between functional csd-PSD alleles are given in Table 3. Notably, when the numbers of amino acid differences excluding the HVR were compared among these pairs, we found five (out of 14) pairs with no amino acid differences in the HVR flanking region, but with changes within the HVR ranging from 2 to 17, providing evidence for the importance of the HVR in determining csd specificities.

Combined, our comparative sequence analyses of $c s d$ exons 6-9, csd-PSD and the neutral loci suggest that very few (1-2) nonsynonymous amino acid changes, basically located within the HVR, are sufficient for generating new functional csd alleles in A. florea.

\section{Evolutionary history of sex-determining genes in A. florea}

To explore the evolutionary history of $A f-c s d$ and Af-fem genes, we compared their genealogical relationship to other Apis species using a subset of the examined alleles (Figure 3). The csd alleles fall into separate clades according to their species origin, which is supported by high bootstrap values $(>99)$. No trans-specific allelic pattern appeared
Table 3 Synonymous $\left(d_{S}\right)$ and nonsynonymous $\left(d_{N}\right)$ differences per site and number of amino acid differences in functional pairs of csd-PSD alleles

\begin{tabular}{|c|c|c|c|c|c|c|}
\hline Allele 1 & Allele 2 & $d_{s}$ & s.e. & $\mathrm{d}_{n}$ & s.e. & Amino acid differences \\
\hline P5I2.1 & P512.3 & 0.009 & 0.009 & 0.003 & 0.003 & $1(10)$ \\
\hline P4I1.1 & P4I1.2 & 0.000 & 0.000 & 0.000 & 0.000 & $0(13)$ \\
\hline P5I1.1 & P5I1.3 & 0.000 & 0.000 & 0.000 & 0.000 & $0(2)$ \\
\hline P1I1.1 & P1I1.3 & 0.000 & 0.000 & 0.006 & 0.004 & $2(5)$ \\
\hline P12।1.1 & P12I1.4 & 0.009 & 0.009 & 0.003 & 0.003 & $1(8)$ \\
\hline P3I1.3 & P3।1.4 & 0.000 & 0.000 & 0.006 & 0.004 & $2(8)$ \\
\hline $\mathrm{P} 1 \mathrm{I} 2.1$ & $\mathrm{P} 1 \mathrm{I} 2.2$ & 0.000 & 0.000 & 0.003 & 0.003 & $1(1)$ \\
\hline P7I1.1 & P7I1.2 & 0.000 & 0.000 & 0.003 & 0.003 & $1(1)$ \\
\hline P7I2.1 & P7I2.6 & 0.000 & 0.000 & 0.006 & 0.004 & $0(5)$ \\
\hline P9I1.3 & P9I1.4 & 0.000 & 0.000 & 0.000 & 0.000 & $0(6)$ \\
\hline P10I1.1 & P10I1.2 & 0.000 & 0.000 & 0.003 & 0.003 & $1(1)$ \\
\hline P3I2.1 & P3I2.3 & 0.000 & 0.000 & 0.003 & 0.003 & $1(1)$ \\
\hline P6I2.1 & P6I2.2 & 0.000 & 0.000 & 0.006 & 0.004 & $0(17)$ \\
\hline P8I1.1 & P8I1.1 & 0.000 & 0.000 & 0.003 & 0.003 & $1(3)$ \\
\hline
\end{tabular}

The hypervariable region (HVR) was excluded in the analysis. Number of amino acid differences including the HVR are given in parenthesis.

in the genealogy as a result of adding the A. florea data, which is consistent with the findings of the previous studies from 2008. Next we applied the Branch-Site REL model (see Materials and methods section) to test for an excess of nonsynonymous over synonymous substitutions along phylogenetic branches. We used this model to circumvent the problem, where with one foreground branch and treating other branches as background leads to an exclusion of important sites, reducing the statistical power of the likelihood ratio tests, as would happen in branch-site two tests.

As the Branch-Site REL method uses unrestricted combinations of selective regimes across sites and branches, we detected different branches with significant signs of episodic diversifying selection for $A$. mellifera $\left(\mathrm{a}^{*}\right)$, A. dorsata $\left(\mathrm{b}^{*}\right)$ and A. cerana $\left(\mathrm{c}^{*}\right)$, whereas for A. florea, these signs were not detected $\left(\mathrm{d}^{\star}, P=0.5\right.$; Figure 4 and Table 4$)$. The branch leading to the fem sequences $\left(\mathrm{e}^{*}\right)$ displays signs of purifying selection, as known from previous studies. Interestingly, the most basal csd branch $\left(\mathrm{g}^{*}\right)$ exhibits a rather weak $\left(d_{\mathrm{n}} / d_{\mathrm{s}}=0.6\right)$ but significant $(P<0.001)$ sign of diversifying selection, likely influenced by the contrasting evolutionary forces acting on $c s d$ alleles in A. florea and the other Apis species.

\section{DISCUSSION}

Evolutionary differences and functional conservation of the $c s d / f e m$ gene complex in Apis

The identification and analyses of the sex-determining genes csd and fem in A. florea broaden our understanding of the evolutionary dynamic of sex determination in the Apis lineage. We found marked differences in the number of polymorphisms within csd alleles (Table 1) and in Csd protein regions in A. florea (Figure 2) compared with the three bee species $A$. mellifera, A. cerana and A. dorsata. Our results suggest that alongside various evolutionary forces, the underlying biology of the open-nesting $A$. florea may explain the structural and sequence-based differences that exist when compared with other Apis species.

We found evidences that only few amino acid differences between $A$. florea csd alleles seem to be necessary and sufficient to encode functional heterozygotes (Table 3, Figure 2). Considering the five allelic pairs that show no amino acid differences except for those located within the HVR (Table 3), we suggest that the HVR alone may 


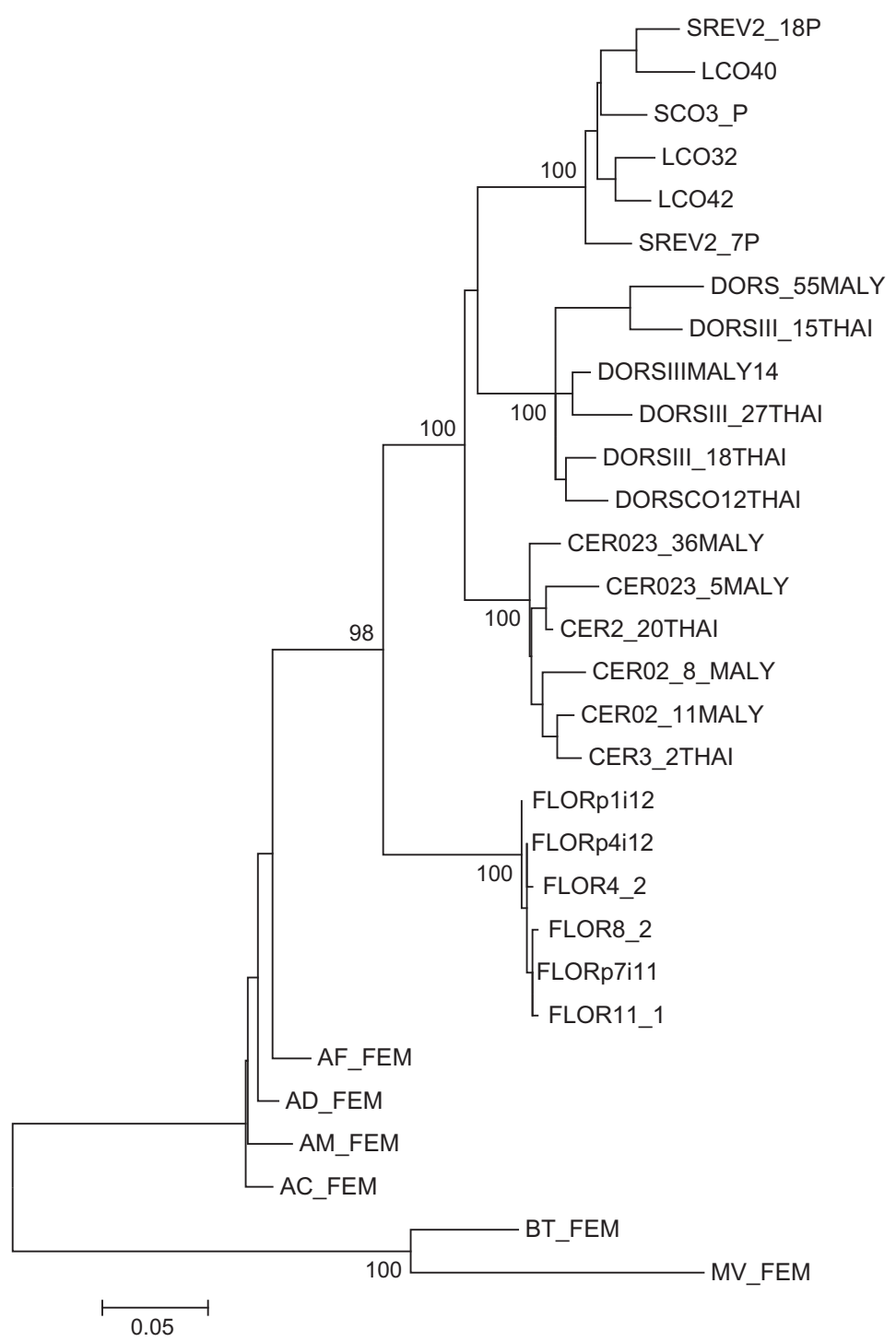

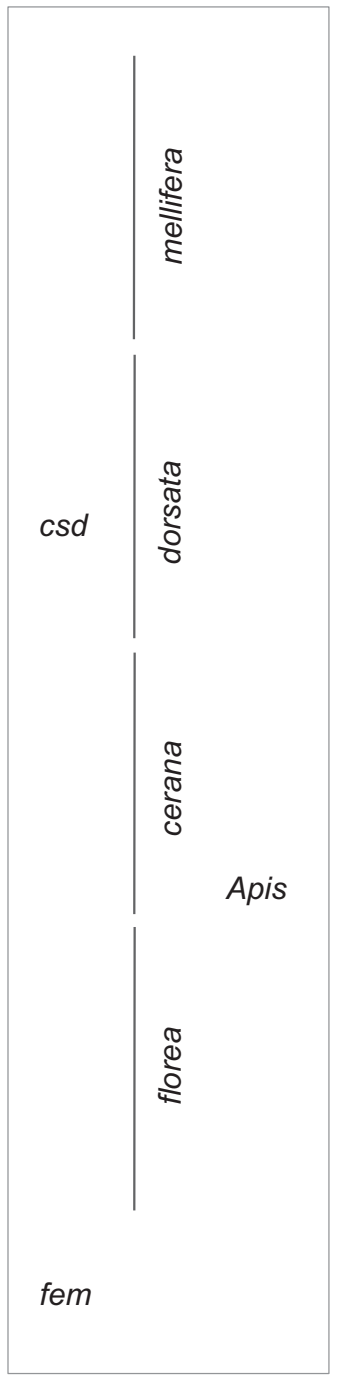

fem B. terrestris

fem M. compressipes

Figure 3 Phylogenetic relationship and substitutions along branches for csd and fem sequences. The evolutionary history was inferred using maximum likelihood based on the Kimura-2 parameter model. The tree with the highest log-likelihood $(-1631.25)$ is shown. The percentage of replicate trees in which the associated taxa clustered together in the bootstrap test (1000 replicates) is shown next to the branches. The evolutionary distances are given in units of the number of nucleotide substitutions per site. A discrete Gamma distribution was used to model the evolutionary rate differences between sites (two categories $(+G$, parameter $=1.63)$ ). The analysis involved 30 nucleotide sequences. The codon positions included were first+second. All positions containing gaps were eliminated. There were a total of 232 positions in the final data set.

encode allelic specificity to initiate female development. Although we have not analyzed the full-length coding sequence of csd for these specific allele pairs, based on the low diversity in the other exons (Table 1), it seems unlikely that substantially more amino acid changes have accumulated in the regions other than the PSD region of Csd. The analysis of functionally different $A$. mellifera csd alleles indicates that on an average, no reduced variability to the extent found for $A$. florea exists within $A$. mellifera $c s d$, as they are an order of magnitude more diverse $\left(\pi_{\text {Amell_csd }}=0.07\right.$ vs $\left.\pi_{\text {Aflor_csd }}=0.007\right)$. Therefore, the appearance of low-diversity alleles in A. florea seems more the rule than the exception when compared with other Apis species.

Based on the amino acid composition within the HVR, asparagine $(\mathrm{N})$ is the predominating residue. No repeated motifs composed of other amino acid residues, as have been observed for the HVR of other Apis species (for example, $\left((\mathrm{N})_{1-5} / \mathrm{Y}\right)$ or $\left.(\mathrm{KHYN})_{1-4}\right) \mathrm{KH}$ ), were found in A. florea csd. However, single serine residues ( $\mathrm{S}$ ) are consistently found at variable positions in pairs of functionally different $c s d-P S D$ alleles obtained from female individuals, indicating an as yet unknown pattern of these serines within the HVR. Serine is commonly phosphorylated and exhibits binding properties, including the capacity to establish stable protein structures (Betts and Russell, 2003). Thus, it may be speculated that the formation of the Csd protein leads to structures in the HVR that can directly mediate csd allelic specificities in A. florea. Support for this hypothesis is given by a csd allele pair in the study of A. mellifera (Beye et al., 2013) containing three amino acid differences within the HVR only that trigger female development. In 


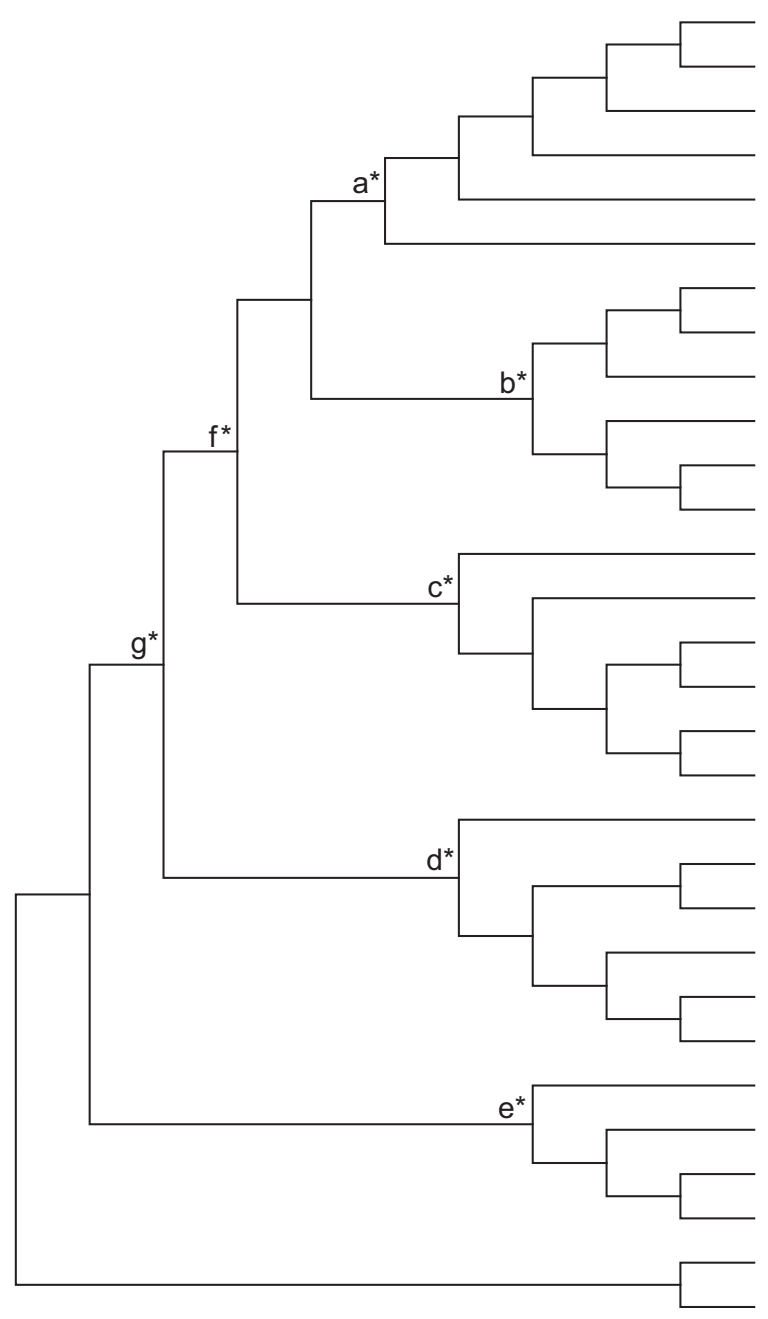

A. mellifera

A. dorsata

csd

A. cerana

A. florea

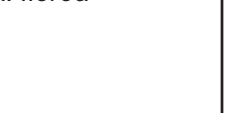

A. florea

A. mellifera

A. cerana

A. dorsata

B. terrestris

M. compressipes

fem

Figure 4 Topology representing fem and $c s d$ evolution in Apis. Marks $\mathrm{a}^{*}-\mathrm{g}^{*}$ represent the branches leading to the different clades of Apis (a*- $\mathrm{d}^{*}$ ) and the internal branches ( $f^{*}$ and $g^{*}$ ) within $c s d$. $e^{*}$ marks the branch of Apis-fem. Strong signs of diversifying selection are given for $a^{*}-c^{*}$ but not for $d^{*}$ (see Table 4 for corresponding results of the Branch-Site REL model. Fem $\left({ }^{*} \mathrm{e}\right)$ shows strong signs of purifying selection.

Table 4 Summary of branches (as marked in Figure 4) under episodic diversifying selection identified by the random effects Branch-Site REL model

\begin{tabular}{lccc}
\hline Branch & Mean $\mathrm{d}_{N} / \mathrm{d}_{S}$ & LRT & P-value \\
\hline $\mathrm{a}^{*}$ & 4.758 & 5.73 & 0.008 \\
$\mathrm{~b}^{*}$ & 3.662 & 10.85 & $4.92 \mathrm{E}-04$ \\
$\mathrm{c}^{*}$ & 10 & 15.55 & $4.02 \mathrm{E}-05$ \\
$\mathrm{~d}^{*}$ & 0.201 & -0.73 & 0.5 \\
$\mathrm{e}^{*}$ & 0 & 0 & 1 \\
$\mathrm{f}^{*}$ & 1.46 & 0.42 & 0.26 \\
$\mathrm{~g}^{*}$ & 0.61 & 10.98 & $4.61 \mathrm{E}-04$ \\
\hline
\end{tabular}

Likelihood Ratio test (LRT) and statistical significance were obtained by using the HyPhy package.

addition, the interaction of proteins produced from different $c s d$ alleles in A. florea may be altered by modification of the coiled-coil motif compared with other Apis species (Figures $2 \mathrm{~b}$ and $\mathrm{c}$ ). The rise of functional protein domains and structures known to be ubiquitous is documented as being rapid and diverse in manner (Ponting and Russell, 2002). Therefore, a coiled-coil motif may likely change in composition and position in orthologs among species but yet maintain its function. We detected a coiled-coil motif in the $\mathrm{N}$-terminal region of Af-Csd (Figure 1a, marked with a triangle) and in the corresponding region in Af-Fem (up to $98 \%$ probability), but not within the csd-PSD region of A. florea (Figure 2). Our analysis indicates that in the other Apis species, no such coiled-coil can be formed in the corresponding regions of $A f-$ Csd and Af-Fem. This motif follows the principle of a 7-11-7 coiled-coil motif (the formation of helices in which seven residues flank eleven residues), which contrasts with the motif located within the PSD in other Apis species, for which a 4-3-4 type (four residues flank a three residue unit in a helical formation) has been described, which could lead to an altered protein-binding behavior like binding strength or association (Lupas et al, 1991; Hicks et al, 1997).

The analyses of nucleotide polymorphisms and their frequency spectrum indicate various evolutionary forces acting on A. florea csd. We detected low-average nucleotide diversity for both nonsynonymous and synonymous sites, whereas the ratio of $\pi_{\mathrm{N}} / \pi_{\mathrm{S}}=1.6$ indicates adaptive evolution acting within the region of exons $6+7$. This is supported by negative values of Tajima's $D$ and Fay and Wu's H-statistic, and a high ratio of $\pi_{\mathrm{N}} / \pi_{\mathrm{S}}$, suggesting positive selection in this particular region, known to be a common target of selection and a PSD (Hasselmann et al, 
2008b). The remarkably low synonymous diversity on csd that equals the one calculated for noncoding loci scattered within the genome indicates that the effect of balancing selection observed in csd-PSD of other Apis (elevated $\pi_{\mathrm{s}}$ ) is not pronounced in A. florea. The frequency spectrum of polymorphic sites in our data suggest that demographic effects may have acted in the recent history of $A$. florea, which could have erased possible signs of balancing selection (for example, increased $\pi_{\mathrm{s}}$ values). Strengthened by our analyses (Table 1), a possible explanation is a recent population growth after a bottleneck.

Bottlenecks might be a frequent occurring phenomenon in A. florea, as this species, originally endemic in South East Asia, is now expanding westward, present now in the Middle East, the Arabian Peninsula and invading Israel and the Sudan (Moritz et al, 2010; Hepburn, 2011). Thus, one may expect that over the course of this long-range expansion, the populations have passed several bottlenecks in the past, raising an interesting issue for future research regarding our sampling, which was restricted to the south of Thailand. In addition, in A. florea, the phenomenon of nest abandonment by swarming and migration is known to be ubiquitous (Hepburn, 2011), as their nests are freely exposed to the environment (open-air nesting). By contrast, the cavity-nesting A. mellifera do not show this frequent and strong fluctuation in population size. In addition to this behavior of abandonment and rebuilding of colonies, $A$. florea queens mate with few (6-10) males, which is in strong contrast to A. mellifera queens (mating with up to 25 males) or $A$. dorsata queens ( $>40$ matings), also known to be open-air nesting (Oldroyd et al, 1995; Kraus et al, 2005). This species-specific behavior may act as a major force leading to a repeating reduction in the effective population size, explaining the biased nucleotide-frequency spectrum.

It seems reasonable to assume that due to the extraordinary advantage of heterozygosity at the csd locus, single mutations are selectively favored in a newly founded population. Fay and Wu's Hstatistic, known to be very sensitive for selective sweeps, detected such signs when applied to Af-csd exons 6-9, but we also see indications for population size expansion after a bottleneck in our data (Table 1). Our previous studies (Hasselmann et al, 2008b; Lechner et al, 2014) examined genetic drift in Apis species and high-origination rates, in A. mellifera specifically, as the major drivers for csd allele evolution, leading to coalescence times shorter than expected under such a selective regime of balancing selection. Interestingly, in the early history of Apis evolution, A. florea seems to combine both in a most pronounced way.

We found differences in signs of selection along the phylogenetic branches for csd in A. florea compared with other Apis species (Figure 4). Whereas strong and significant signs of positive selection were found for the csd branches leading to A. mellifera/A. cerana and A. dorsata (Figure 4 and Table 4 ) with $d_{\mathrm{n}} / d_{\mathrm{s}}$ ranging from 3.6 to 10 , this value for $c s d$ in A. florea was not significant (0.2). Thus, although a substantial number of nonsynonymous substitutions have been fixed in the lineage of A. florea, the low number of polymorphisms in the current csd allele data set may limit the detectable signs of selection. To test whether the results for the A. florea branches were influenced by the low-sequence diversity, we used a modified data set of A.mellifera, A. cerana and A.dorsata containing csd sequences more similar to each other and re-ran the Branch-Site REL analyses (Supplementary Table $4)$. The $d_{\mathrm{n}} / d_{\mathrm{s}}$ ratio of the non-florea Apis was thereupon strongly reduced $(0.23-0.56)$ to the level of $A$. florea (0.23), supporting our hypothesis that the limited $c s d$-sequence diversity affects the statistical power of the analysis.
Contrasting evolution in the sex determination pathway among Apis species

As is known for several holometabolic species, primary signals of sex determination pathways show an enormous diversity, whereas downstream targets remain conserved as a transductional core (fem/tra - $d s x$ genes). Furthermore, these primary signals seem to be rapidly evolving, as they derive from different origins such as from gene duplication or allelic variation (Bopp et al, 2014). Among closely related species like Apis and Bombus, gene duplication of fem occurred independently (Koch et al. 2014) and likely led to different functions. Therefore, it is reasonable to argue that the evolution of $A$. florea and A. mellifera/A. cerana/A. dorsata csd headed in different directions, leading to structural and functional modifications between the csd genes for which we found evidence in the current study.

Our results show a modified coiled-coil formation in A. florea Csd compared with the other species caused by three amino acid differences in the N-terminal region and one difference in the C-terminal region of Csd. The N-terminal coiled-coil motif in A. florea seems to have evolved from specific modifications (one substituted and two deleted amino acids) of its precursor Fem (Figure 2c). In addition, few amino acid changes are needed to give rise to new csd specificities, harboring a less complex HVR than in the other Apis species (Figure 2). These findings lead us to two alternative scenarios of $\mathrm{fem} / \mathrm{csd}$ evolution. The first scenario involves a single duplication of fem at the early stage, prior to the divergence of Apis species, with lineage-specific modifications of csd governed by evolutionary constraints. This scenario is supported by high homologies among all Apis-csd, including protein domains, HVR and coil motifs. Nevertheless, marked differences between $A f-c s d$ and remaining Apiscsd suggest an evolutionary transition, from the early stage of the duplication beginning, which has led to a $\mathrm{fem} / \mathrm{cs} d$ complex in A. florea compatible with (or influenced by) its biology. The second scenario would be an independent duplication of fem within the Apis lineage, with one Apis-csd lineage present in A. florea and the other in A. mellifera/A. cerana/A. dorsata, leading to an independent but constrained evolution of $c s d$ as the primary signal of sex determination. Given the high structural similarity of $c s d$ within Apis, irrespective of the evolutionary differences, we conclude this to be a rather unlikely scenario. Our conclusion is further strengthened by the analysis of the fem gene of $A$. andreniformis, a dwarf honey bee closely related to $A$. florea, comprising an identical coiled-coil motif (Hasselmann, unpublished data).

The first scenario is in agreement with the diverse evolutionary fate of gene duplicates during their initial phase. These genes can be altered through randomly occurring mutations, with major consequences for their subsequent evolution (for example, neo-functionalization and pseudogenization; Conant and Wolfe, 2008). Pseudogene sequences and non-coding genomic fragments of csd occurring in the A. mellifera genome, which were non-functional but still had high similarity to $c s d$, were previously identified and interpreted as likely trans-specific alleles (Cho et al, 2006; for discussion see Hasselmann et al, 2008b). In our present study, none of the evolutionary young csd alleles provide indications for being trans-specific. The study of Liu et al. (2011) describes variable csd HVR repeated motifs amplified from A. florea genomic DNA, for which we found no evidence in our RACE and reverse transcription experiments. Thus, the scenario of neofunctionalization of distinct ancestral csd-types in A. florea and in A. mellifera/A. cerana/A. dorsata seems likely. The frequent and independent duplication of fem in Hymenopteran lineages (Geuverink and Beukeboom, 2014; Koch et al, 2014) (Bee10Genome consortium, Kapheim et al, 2015) seems to be a general phenomenon, which 
may be of broader relevance to better understand the evolution of sex determination pathways in other insect species.

In summary, our data suggest that the sex-determining function of csd in A. florea follows the same principle as that proposed for $A$. mellifera, whereas the molecular mechanism of csd seems to be encoded by fewer amino acid differences, a less complex HVR and more modified coiled-coil motifs than that described for A. mellifera. Moreover, csd allele evolution seems to be heavily influenced by the biology of A. florea. The resulting strong impact of genetic drift and bottleneck events is also of pronounced interest for the conservation of other (for example, solitary) bee species.

\section{DATA ARCHIVING}

Sequence data available from GenBank: accession numbers KS297794KS297876 (A. florea fem), KS297743-KS297793 (A. florea csd).

\section{CONFLICT OF INTEREST}

The authors declare no conflict of interest.

\section{ACKNOWLEDGEMENTS}

We are very thankful to Jochen Pflugfelder for collecting A. florea samples and three anonymous reviewers for their helpful comments. This work was supported by grants from the Deutsche Forschungsgemeinschaft (HA 5499/3-1 and HA 5499/3-2 to MH).

Betts MJ, Russell RB (2003). Amino acid properties and consequences of subsitutions. In: Barnes MR, Gray IC (eds). Bioinformatics for Geneticists. Wiley: West Sussex.

Beye M, Gattermeier I, Hasselmann M, Gempe T, Schioett M, Baines JF et al. (2006). Exceptionally high levels of recombination across the honey bee genome. Genome Res 16: 1339-1344.

Beye M, Hasselmann M, Fondrk MK, Page Jr RE, Omholt SW (2003). The gene csd is the primary signal for sexual development in the honey bee and encodes a SR-type protein. Cell 114: 419-429.

Beye M, Seelmann C, Gempe T, Hasselmann M, Vekemans X, Fondrk MK et al. (2013). Gradual Molecular Evolution of a Sex Determination Switch through Incomplete Penetrance of Femaleness. Current Biology 23: 2559-2564.

Bopp D, Saccone G, Beye M (2014). Sex determination in insects: variations on a common theme. Sex Dev 8: 20-28.

Cho S, Huang ZY, Green DR, Smith DR, Zhang J (2006). Evolution of the complementary sex-determination gene of honey bees: balancing selection and trans-species polymorphisms. Genome Res 16: 1366-1375.

Conant GC, Wolfe KH (2008). Turning a hobby into a job: how duplicated genes find new functions. Nat Rev Genet 9: 938-950.

Fay JC, Wu Cl (2000). Hitchhiking under positive Darwinian selection. Genetics 155: $1405-1413$.

Fu YX (1997). Statistical tests of neutrality of mutations against population growth, hitchhiking and background selection. Genetics 147: 915-925.

Gempe T, Hasselmann M, Schioett M, Hause G, Otte M, Beye M (2009). Sex determination in honeybees: Two separate mechanisms induce and maintain the female pathway. PLOS Biol 7: e1000222.

Geuverink E, Beukeboom LW (2014). Phylogenetic distribution and evolutionary dynamics of the sex determination genes doublesex and transformer in insects. Sex Dev 8: 38-49.

Hasselmann M, Beye M (2004). Signatures of selection among sex-determining alleles of the honey bee. Proc Natl Acad Sci USA 101: 4888-4893.

Hasselmann M, Gempe T, Schiott M, Nunes-Silva CG, Otte M, Beye M (2008a). Evidence for the evolutionary nascence of a novel sex determination pathway in honeybees. Nature 454: 519-522.

Hasselmann M, Lechner S, Schulte C, Beye M (2010). Origin of a function by tandem gene duplication limits the evolutionary capability of its sister copy. Proc Natl Acad Sci USA 107: $13378-13383$
Hasselmann M, Vekemans X, Pflugfelder J, Koeniger N, Koeniger G, Tingek S et al. (2008b). Evidence for convergent nucleotide evolution and high allelic turnover rates at the complementary sex determiner gene of western and asian honeybees. Mol Biol Evol 25: 696-708.

Hepburn HR Absconding, migration and swarming. Hepburn HR, Radloff S. (2011) Honeybees of Asia. Springer: Berlin, Heidelberg, 133-158.

Hicks MR, Holberton DV, Kowalczyk C, Woolfson DN (1997). Coiled-coil assembly by peptides with non-heptad sequence motifs. Fold Des 2: 149-158.

Hudson RR, Kaplan NL (1985). Statistical properties of the number of recombination events in the history of a sample of DNA sequences. Genetics 111: 147-164.

Hudson RR, Kreitman M, Aguade M (1987). A test of neutral molecular evolution based on nucleotide data. Genetics 116: 153-159.

Innan H, Kondrashov F (2010). The evolution of gene duplications: classifying and distinguishing between models. Nat Rev Genet 11: 97-108.

Kapheim KM, Pan H, Li C, Salzberg SL, Puiu D, Magoc T et al. (2015). Genomic signatures of evolutionary transitions from solitary to group living. Science 348: 1139-1143.

Koch V, Nissen I, Schmitt BD, Beye M (2014). Independent evolutionary origin of fem paralogous genes and complementary sex determination in hymenopteran insects. PLoS One 9: e91883.

Kosakovsky Pond SL, Frost SD, Muse SV (2005). HyPhy: hypothesis testing using phylogenies. Bioinformatics 21: 676-679.

Kosakovsky Pond SL, Murrell B, Fourment M, Frost SDW, Delport W, Scheffler K (2011). A random effects branch-site model for detecting episodic diversifying selection. Mol Biol Evol 28: 3033-3043.

Kraus FB, Neumann P, Moritz RFA (2005). Genetic variance of mating frequency in the honeybee (Apis mellifera L.). Insectes Soc 52: 1-5.

Lechner S, Ferretti L, Schoning C, Kinuthia W, Willemsen D, Hasselmann M (2014). Nucleotide variability at its limit? insights into the number and evolutionary dynamics of the sex-determining specificities of the honey bee Apis mellifera. Mol Biol Evol 31: 272-287.

Librado P, Rozas J (2009). DnaSP v5: a software for comprehensive analysis of DNA polymorphism data. Bioinformatics 25: 1451-1452.

Liu ZY, Wang ZL, Wu XB, Yan WY, Zeng ZJ (2011). csd alleles in the red dwarf honey bee (Apis florea, Hymenoptera: Apidae) show exceptionally high nucleotide diversity. Insect Sci 18: 645-651.

Lo N, Gloag RS, Anderson DL, Oldroyd BP (2010). A molecular phylogeny of the genus Apis suggests that the Giant Honey Bee of the Philippines, A-breviligula Maa, and the Plains Honey Bee of southern India, A-indica Fabricius, are valid species. Syst Entomol 35: 226-233.

Lupas A, Van Dyke M, Stock J (1991). Predicting coiled coils from protein sequences. Science 252: 1162-1164.

Nissen I, Muller M, Beye M (2012). The Am-tra2 gene is an essential regulator of female splice regulation at two levels of the sex determination hierarchy of the honeybee. Genetics 192: 1015-1026.

Oldroyd BP, Smolenski AJ, Cornuet JM, Wongsiri S, Estoup A, Rinderer TE et al. (1995). Levels of polyandry and intracolonial genetic-relationships in Apis-Florea. Behav Ecol Sociobiol 37: 329-335.

Palmer KA, Oldroyd BP (2000). Evolution of multiple mating in the genus Apis. Apidologie 31: 235-248.

Ponting CP, Russell RR (2002). The natural history of protein domains. Annu Rev Bioph Biom 31: 45-71.

Ramirez SR, Nieh JC, Quental TB, Roubik DW, Imperatriz-Fonseca VL, Pierce NE (2010). A molecular phylogeny of the stingless bee genus Melipona (Hymenoptera: Apidae). Mol Phylogenet Evol 56: 519-525.

Schmieder S, Colinet D, Poirie M (2012). Tracing back the nascence of a new sexdetermination pathway to the ancestor of bees and ants. Nat Commun 3: 895.

Tajima $\mathrm{F}$ (1989). The effect of change in population size on DNA polymorphism. Genetics 123: $597-601$.

Takahata N (1990). A simple genealogical structure of strongly balanced allelic lines and trans-species evolution of polymorphism. Proc Natl Acad Sci USA 87: 2419-2423.

Tamura K, Peterson D, Peterson N, Stecher G, Nei M, Kumar S (2011). MEGA5: Molecular Evolutionary Genetics Analysis using maximum likelihood, evolutionary distance, and maximum parsimony methods. Mol Biol Evol 28: 2731-2739.

Vekemans X, Slatkin M (1994). Gene and allelic genealogies at a gametophytic selfincompatibility locus. Genetics 137: 1157-1165.

Woyke J (1963). Drone larvae from fertilized eggs of the honeybee. Japic Res 2: 19-24.

Yokoyama S, Nei M (1979). Population dynamics of sex determining alleles in honey bees and self-incompatibility in plants. Genetics 91: 609-626.

Supplementary Information accompanies this paper on Heredity website (http://www.nature.com/hdy) 